\title{
On the absolute value of the air-fluorescence yield
}

\author{
J. Rosado*, F. Blanco, F. Arqueros \\ Departamento de Física Atómica, Molecular y Nuclear, Facultad de Ciencias Físicas, \\ Universidad Complutense de Madrid, E-28040 Madrid, Spain
}

\begin{abstract}
The absolute value of the air-fluorescence yield is a key parameter for the energy reconstruction of extensive air showers registered by fluorescence telescopes. In previous publications, we reported a detailed Monte Carlo simulation of the air-fluorescence generation that allowed the theoretical evaluation of this parameter. This simulation has been upgraded in the present work. As a result, we determined an updated absolute value of the fluorescence yield of $7.9 \pm 2.0 \mathrm{ph} / \mathrm{MeV}$ for the band at $337 \mathrm{~nm}$ in dry air at $800 \mathrm{hPa}$ and $293 \mathrm{~K}$, in agreement with experimental values. We have also performed a critical analysis of available absolute measurements of the fluorescence yield with the assistance of our simulation. Corrections have been applied to some measurements to account for a bias in the evaluation of the energy deposition. Possible effects of other experimental aspects have also been discussed. From this analysis, we determined an average fluorescence yield of $7.04 \pm 0.24 \mathrm{ph} / \mathrm{MeV}$ at the above conditions.
\end{abstract}

Keywords: air-fluorescence yield, fluorescence telescopes, ultra-high-energy cosmic rays, extensive air showers

\section{Introduction}

Ultra-high-energy cosmic rays are efficiently detected from the isotropic fluorescence light produced in the atmosphere by the extensive air showers. This technique was used by HiRes [1] and is presently employed by the Pierre Auger Observatory [2] and the Telescope Array experiment [3]. The

\footnotetext{
${ }^{*}$ Corresponding author

Email address: jaime_ros@fis.ucm.es (J. Rosado)
} 
JEM-EUSO Collaboration [4 has also proposed to record the air-fluorescence traces of cosmic rays from the top of the atmosphere in a satellite mission. The number of fluorescence photons emitted at a given shower position is proportional to the energy deposited in the atmosphere; hence, the longitudinal development of the light registered by fluorescence telescopes provides a calorimetric measure of the shower energy. The fluorescence yield (FY) in air, i.e., the number of photons emitted per unit energy deposited in the atmosphere, is thus a key parameter in this technique.

The production of fluorescence light in the atmosphere has been studied since long ago in various fields, e.g., in aurora physics [5]. This light emission is due to the de-excitation of molecules previously excited by charged particles. ${ }^{1}$ In the spectral range of interest in the cosmic-ray field (i.e., around $300-400 \mathrm{~nm}$ ), the air fluorescence is dominated by the second positive (2P) band system of $\mathrm{N}_{2}\left(\mathrm{C}^{3} \Pi_{\mathrm{u}} \rightarrow \mathrm{B}^{3} \Pi_{\mathrm{g}}\right)$ and the first negative (1N) band system of $\mathrm{N}_{2}^{+}\left(\mathrm{B}^{2} \Sigma_{\mathrm{u}}^{+} \rightarrow \mathrm{X}^{2} \Sigma_{\mathrm{g}}^{+}\right)$. Excited species can also relax without emitting light through collisions with other molecules [6]; as a consequence, the fluorescence intensity depends on the atmospheric conditions (i.e., pressure, temperature and humidity).

Although the processes underlying the generation of this radiation are fully understood since the early twentieth century, the associated parameters have not been known with enough precision for an accurate energy reconstruction of air showers until very recently. The most convenient procedure for the analysis of cosmic-ray data is to combine an accurate value of the absolute FY at given air conditions for a reference molecular band (or a wavelength interval) with the relative intensities of the fluorescence spectrum and those parameters related to the atmospheric dependencies. This paper is focused on the absolute value of the FY. Note that the uncertainty in this parameter translates almost linearly to the energy scale of the fluorescence telescopes.

In previous works [7-11], we presented a theoretical evaluation of the FY based on a Monte Carlo (MC) simulation of both the energy deposition and fluorescence emission. It was shown that the nitrogen fluorescence is mostly induced by low-energy secondary electrons generated along the tracks of energetic charged particles. Consequently, our main efforts were directed toward a detailed characterization of the generation and transport of these

\footnotetext{
${ }^{1}$ Strictly speaking, this radiation should be named scintillation instead of fluorescence.
} 
secondary electrons. In this paper, several relevant upgrades of our MC algorithm are described (section 2) and the corresponding updated results are reported (section 3). Details are given in two Appendices.

Several measurements of the absolute FY were carried out in the past years [12 19]. The experimental technique usually consists of a beam of electrons that collides with an air target at known conditions and an appropriate detection system that measures the absolute fluorescence intensity. In [10, 11, we compared the available FY data normalized to common conditions and discussed on the evaluation of the energy deposition in these experiments in comparison with our simulation results. The analysis revealed a full compatibility of the FY measurements as long as the energy deposition in the experimental chamber was accurately determined. This lead us to evaluate an average value of the FY, preliminary results having been shown in unpublished works [20, 21] and in several conferences [22, 24]. Here, we present an update of our analysis including new relevant measurements [18, 19] and some additional considerations (section 4). The procedure to obtain our final result of the average FY and the associated uncertainty is given in section 5 .

\section{Upgrades of the Monte Carlo algorithm}

Our MC algorithm was described in detail in previous publications [7-11] and therefore only a brief overview is given here. The algorithm enables the transport of electrons in the range from $100 \mathrm{GeV}$ down to several $\mathrm{eV}$ inside an interaction region of any geometry filled with nitrogen gas at a selected pressure and temperature. An electron trajectory consists of a succession of "free-flight" steps randomly generated from an exponential distribution with average path length equal to the reciprocal of the product of the gas density and the total cross section. In each step, a discrete random number is generated to select the kind of interaction: elastic, ionization, excitation (without ionization) or bremsstrahlung, the probability of each process being proportional to the corresponding cross section. In elastic interactions, the electron energy does not change but it is scattered in a new random direction according to the differential elastic cross section. In inelastic interactions, the energy lost by the electron and its angular deflection are calculated from appropriate data for the $\mathrm{N}_{2}$ molecule. If the inelastic interaction is an excitation, the average amount of $2 \mathrm{P}$ fluorescence light emitted by the excited nitrogen molecule is evaluated as the ratio of the emission cross section and 
the total excitation cross section. In the case of ionization, the $1 \mathrm{~N}$ fluorescence emitted by $\mathrm{N}_{2}^{+}$is evaluated correspondingly, and a secondary electron is ejected with a random kinetic energy and direction according to the differential ionization cross section and momentum conservation. If the primary electron has enough energy, a K-shell ionization may occur with a probability equal to the ratio of the K-shell ionization cross section and the total ionization cross section, resulting in the emission of a $\mathrm{X}$ ray too $2^{2}$

All the individual interactions of both primary and secondary electrons (and $\mathrm{X}$ rays) are simulated until they either leave the considered interaction region or have an energy below $11 \mathrm{eV}$, which corresponds to the threshold for fluorescence production. Below this threshold, the electron is assumed to deposit its remaining energy in the medium. Bremsstrahlung photons are not tracked, since they are assumed to leave the medium without interacting in it. Note that, unlike many general-purpose MC codes that use the multiple scattering approach, this detailed simulation allows us to describe the energy deposition and the fluorescence emission at a microscopic scale.

We improved some relevant ingredients of our algorithm since the version presented in [11]. The upgrades are described in the following subsections.

\subsection{Energy spectrum of secondary electrons}

An adequate description of the single-differential ionization cross section $\mathrm{d} \sigma_{\text {ion }} / \mathrm{d} W$ of nitrogen as a function of the secondary electron energy $W$ and the incident electron energy $T$ is a key ingredient of our MC algorithm. A simple analytical model of $\mathrm{d} \sigma_{\text {ion }} / \mathrm{d} W$ was presented in [10]; however, exchange effects due to the indistinguishability of the scattered and ejected electrons were neglected. The model has been upgraded to account for these effects as described in Appendix A,

This upgrade results in a slight increase in the production of high-energy secondary electrons, i.e., $\delta$ rays. Although they are very scarce, $\delta$ rays carry a significant fraction of the energy lost by the primary particle and, due to their relatively large ranges, they can leave the field of view of the detector measuring the fluorescence light in a laboratory experiment. As a consequence of this upgrade, the updated results of the energy deposition in a finite medium (see section 3.1) are somewhat lower compared to previous

\footnotetext{
${ }^{2}$ The generation and transport of $\mathrm{X}$ rays were included in [11, but not in earlier versions of the algorithm.
} 
versions of the algorithm. On the other hand, our theoretical predictions of the FY (section 3.2) are almost unaffected, because $\delta$ rays have an efficiency for fluorescence production similar to that of the primary electron.

\subsection{Density-effect correction to the K-shell ionization cross section}

At very high energies $(T \gtrsim 1 \mathrm{GeV})$, the scattering cross sections have to be corrected for the so-called "density effect", which is due to the polarization of the medium [25. In our algorithm, this correction is applied to the total ionization cross section as described in [8], but it was formerly neglected for the (partial) K-shell ionization cross section. In the present version of the algorithm, the density-effect correction has been included in this cross section assuming the same correction term as that used in the total ionization cross section. This assumption is justified by the fact that differences between the energies of the $\mathrm{N}_{2}$ shells are negligible compared to the incident electron energies for which this effect is important.

This upgrade somewhat affects the yield of $\mathrm{X}$ rays, which are responsible for a significant fraction of the energy deposition and the fluorescence emission. There is no significant effect on both the total energy loss and the FY, however, the updated results of the energy deposition in a finite medium for primary electron energies in the GeV range (see subsection 3.1) are slightly lower than those reported in [11].

\subsection{Angular distribution of scattered electrons}

The angular deflections of electron trajectories in air or nitrogen are mainly determined by elastic collisions, although inelastic interactions may also be important. In the present version of our MC algorithm, we have upgraded the the angular distribution of scattered electrons in elastic collisions as described in Appendix B.2. The improvement with respect to previous versions is only relevant for low-energy electrons, which have very short ranges at atmospheric pressure. Therefore, this has no significant impact for the purposes of this paper, although it may be important at very low-pressure conditions, where an accurate tracking of low-energy secondary electrons is necessary [21, 26].

In previous versions of the $\mathrm{MC}$ algorithm, the angular distribution of scattered electrons in inelastic collisions was assumed to be the same as the one used for elastic collisions. This crude approximation was justified by the fact that the final results are only weakly dependent on the details in the tracking of both primary and secondary electrons. However, we realized 
that our algorithm slightly overestimated the average path length of primary electrons of low energies $(T \lesssim 1 \mathrm{MeV})$, resulting in a small but systematic bias on the calculated energy deposition [23]. To correct this bias, the angular distribution of scattered electrons in ionization collisions has been revised as described in Appendix B.1. The sampling of the direction of ejection of secondary electrons is also described in that Appendix.

\section{Updated simulation results}

In general, we are interested in the evaluation of the energy deposition and the fluorescence emission upon the passage of energetic electrons through a given volume of nitrogen or air. Our MC algorithm calculates both magnitudes as the sum of all the tiny contributions of the individual inelastic collisions undergone by the primary electrons as well as by every secondary electron and X ray generated inside the interaction region. The FY is determined as the ratio of the total fluorescence intensity and energy deposition in the same volume. Only the emissions in two reference bands at $337 \mathrm{~nm}$ and $391 \mathrm{~nm}$ of the $2 \mathrm{P}$ and $1 \mathrm{~N}$ systems, respectively, are evaluated, since the relative intensities of the remaining bands in the spectral range of interest are known. The algorithm is for pure nitrogen and it does not include collisional quenching.

As described in previous works [7, 8, 10], we developed a general-purpose simulation, hereafter referred to as "generic simulation", where the primary electrons are not transported. Instead, they are assumed to have a single inelastic interaction at a given point and the ejected secondary particles are tracked up to a certain radial distance $R$. In this way, we obtained both the mean energy deposition and the mean fluorescence emission per primary inelastic interaction. Updated results are presented in subsections 3.1 and 3.2 , respectively.

In addition, realistic simulations including the tracking of primary electrons were carried out for specific geometries. The corresponding FY results are compared with those of the generic simulation in subsection 3.2 .

Finally, the theoretical absolute value of the FY for the $337 \mathrm{~nm}$ band in air at atmospheric conditions was obtained by applying the appropriate quenching reduction. The result is shown subsection 3.3 . 


\subsection{Stopping power and energy deposition}

An important requisite of our MC algorithm is to account for the collision stopping power, that is, the mean energy loss in inelastic collisions per unit mass thickness traversed by an electron. As described in [10], this parameter is related to the molecular parameters used in the algorithm (i.e., excitation and ionization cross sections, mean energy of ejected secondary electrons, etc.) by a simple expression and therefore it can be calculated without the need of a simulation. In figure 1, the result of this calculation is compared with the Bethe-Bloch formula of the stopping power evaluated from the parameterization given by [27]. Results agree within the uncertainties of this parameterization, which were estimated by the authors to be around $1 \%$ for energies above $100 \mathrm{keV}$, but larger at lower energies because of the omission of the shell correction. As discussed in [27, this parametrization of the BetheBloch formula is expected to overestimate the stopping power by about $10 \%$ at $1 \mathrm{keV}$ for low- $Z$ materials and it is no longer valid at lower energies. Our calculations, which are free from this systematic error, give a stopping power lower than that of [27] by $3 \%$ at $10 \mathrm{keV}$ and by $8 \%$ at $1 \mathrm{keV}$, within expectations. This allows us to cover the vast energy range from $100 \mathrm{GeV}$ down to $10 \mathrm{eV}$. Note that an adequate description of the slowdown of low-energy secondary electrons is mandatory for a theoretical evaluation of the FY.

A fraction of the energy lost by the primary electrons is carried away by $\delta$ rays that can escape the interaction region. Therefore, the energy deposition in the medium is lower than the total energy loss, unless the primary electron energy is not high enough to produce $\delta$ rays or the interaction region is very large. Results of our generic simulation for a nitrogen density of $1.137 \mathrm{mg} / \mathrm{cm}^{3}$ (i.e., 1 atm and $300 \mathrm{~K}$ ) and various $R$ values are shown in figure 1. Note that the mean energy deposition per primary interaction provided by this simulation has to be divided by the product of the density and the mean free path of the primary electrons to obtain the differential energy deposition per unit mass thickness $\mathrm{d} E_{\mathrm{dep}} / \mathrm{d} X$. We obtained that, for a typical cm-sized interaction region, the energy deposition is about $10 \%$ lower than the energy loss for primary electrons of $1 \mathrm{MeV}$, and the difference amounts to more than $25 \%$ in the GeV range. We also found that the energy deposition is only weakly dependent on the size of the interaction volume (or the nitrogen density). Therefore, a reasonable accuracy in the energy deposition can be achieved without the need to simulate the fine geometrical details of an experiment.

At usual incident electron energies ( $\gtrsim 10 \mathrm{keV})$, the stopping powers of 


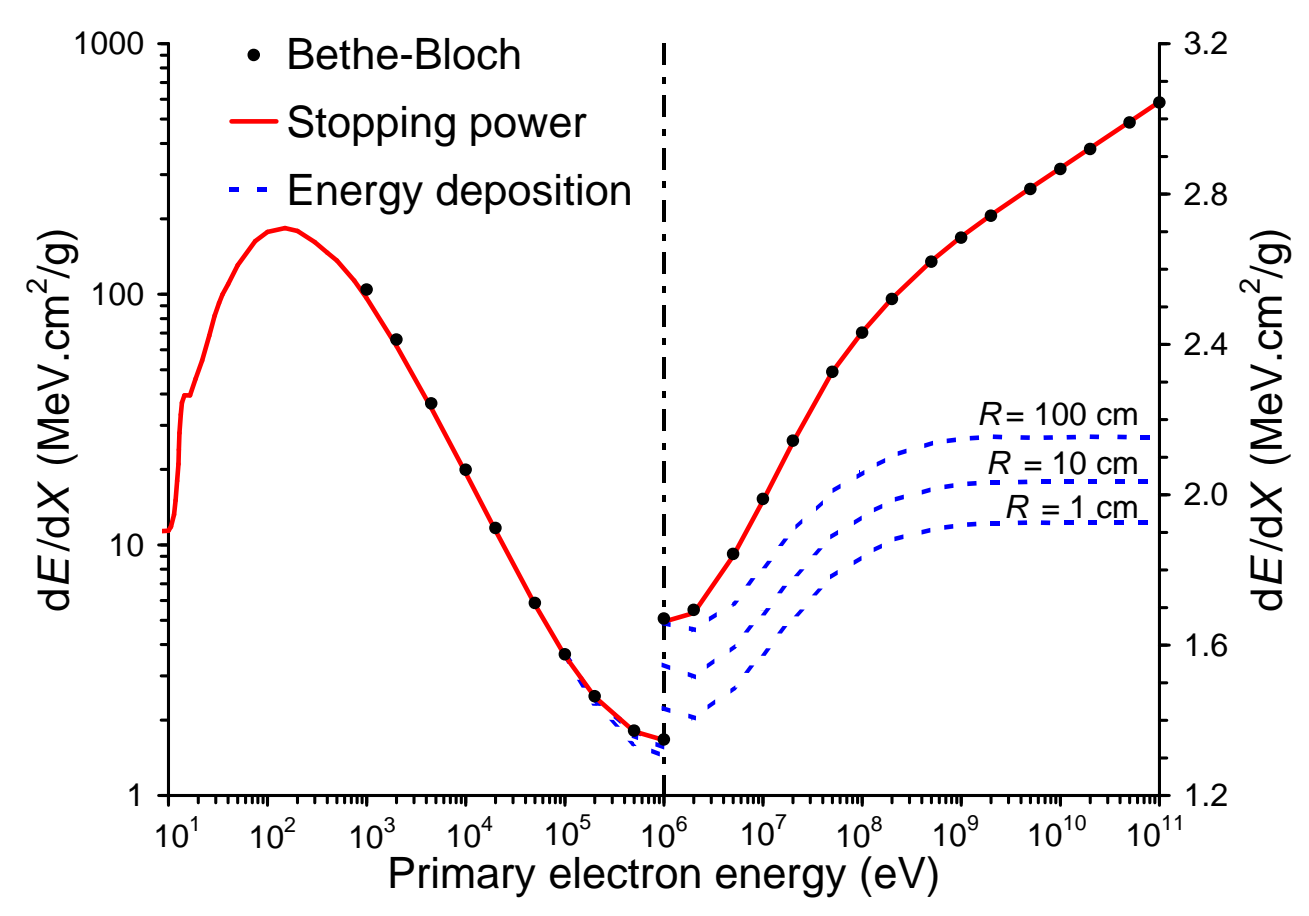

Figure 1: Updated results of collision energy loss and energy deposition per unit mass thickness of nitrogen at $1.137 \mathrm{mg} / \mathrm{cm}^{3}$ (i.e., $1 \mathrm{~atm}$ and $300 \mathrm{~K}$ ). Filled circles: BetheBloch stopping power [27. Solid line (red): stopping power according to the microscopic treatment implemented in our MC algorithm (see text). Dotted lines (blue): $\mathrm{MC}$ results of energy deposition restricted to spheres of radii $R=1,10$ and $100 \mathrm{~cm}$ around the primary interaction point (see text). Different vertical scales are used to represent results for energies below $1 \mathrm{MeV}$ (left axis) and above $1 \mathrm{MeV}$ (right axis).

nitrogen and air are almost identical (within 1\%) when they are expressed in units of mass thickness $3^{3}$ Also, the fraction of energy carried away by $\delta$ rays that leave the interaction volume is expected to be basically the same in both gases at given density. Therefore, the above results of energy deposition in nitrogen are also valid for air.

It was shown in 23] that our updated results of energy deposition, in-

\footnotetext{
${ }^{3}$ The stopping power expressed in these units is basically proportional to the ratio of the atomic number and the atomic weight of the material, and this ratio is equal to $1 / 2$ for both nitrogen and oxygen gases, which constitute $99 \%$ of air.
} 
cluding their dependencies on energy and pressure, are in excellent agreement with those obtained with Geant4 [28]. From this comparison, we estimated an uncertainty of $2 \%$ in the evaluation of the energy deposition at usual conditions of experiments measuring the FY.

\subsection{Nitrogen-fluorescence yield in the absence of quenching}

Simulation results of the FY for the bands at $337 \mathrm{~nm}$ and $391 \mathrm{~nm}$ in nitrogen at $1.137 \mathrm{mg} / \mathrm{cm}^{3}$ in the absence of collisional quenching are shown in figure 2. Our generic simulation predicts that the FY is nearly constant at usual experimental conditions, that is, the fluorescence intensity is basically proportional to the energy deposition in a given volume. Variations in the calculated FY are less than $0.2 \%$ at energies above $100 \mathrm{MeV}$, although a weak energy dependence is found at lower energies. When the electron energy decreases from $100 \mathrm{MeV}$ down to $1 \mathrm{MeV}(1 \mathrm{keV})$, the FY for the $337 \mathrm{~nm}$ band increases by about $2 \%$ (7\%) and that for the $391 \mathrm{~nm}$ band decreases by $0.6 \%$ $(2.5 \%){ }^{7}$ Our calculations also show a very slight dependence on the size of the interaction volume, with variations of less than $1 \%$ for the considered range of $R$ values. These updated FY results are somewhat greater than those previously presented in [10], where the contribution of $\mathrm{X}$ rays to the fluorescence emission had been neglected.

In practice, the FY is measured as the ratio of the total fluorescence emission and the total energy deposited by the electron beam in a nitrogen volume of given geometry. The above-mentioned realistic simulations including the tracking of the primary electrons allow us to evaluate the FY under these conditions. In particular, they account for the fact that the energy of the primary electrons varies along their trajectories. Results for an electron beam crossing a sphere of radius $R=5 \mathrm{~cm}$ are shown in the figure as a function of the incident electron energy. At high energies $(\gtrsim 1 \mathrm{MeV})$, electrons cross the sphere without losing a significant fraction of energy and the FY is fully consistent with that obtained from the generic simulation. Electrons with energies below $50 \mathrm{keV}$ stop completely in the sphere, resulting in slightly steeper variation in the FY with energy. For instance, the FY for

\footnotetext{
${ }^{4}$ The contribution to the energy deposited by an extensive air shower from electrons with energy lower than $1 \mathrm{MeV}$ is only of about $22 \%$ 29. Therefore, this weak energy dependence of the FY has no relevant impact on the calibration of the fluorescence telescopes.
} 


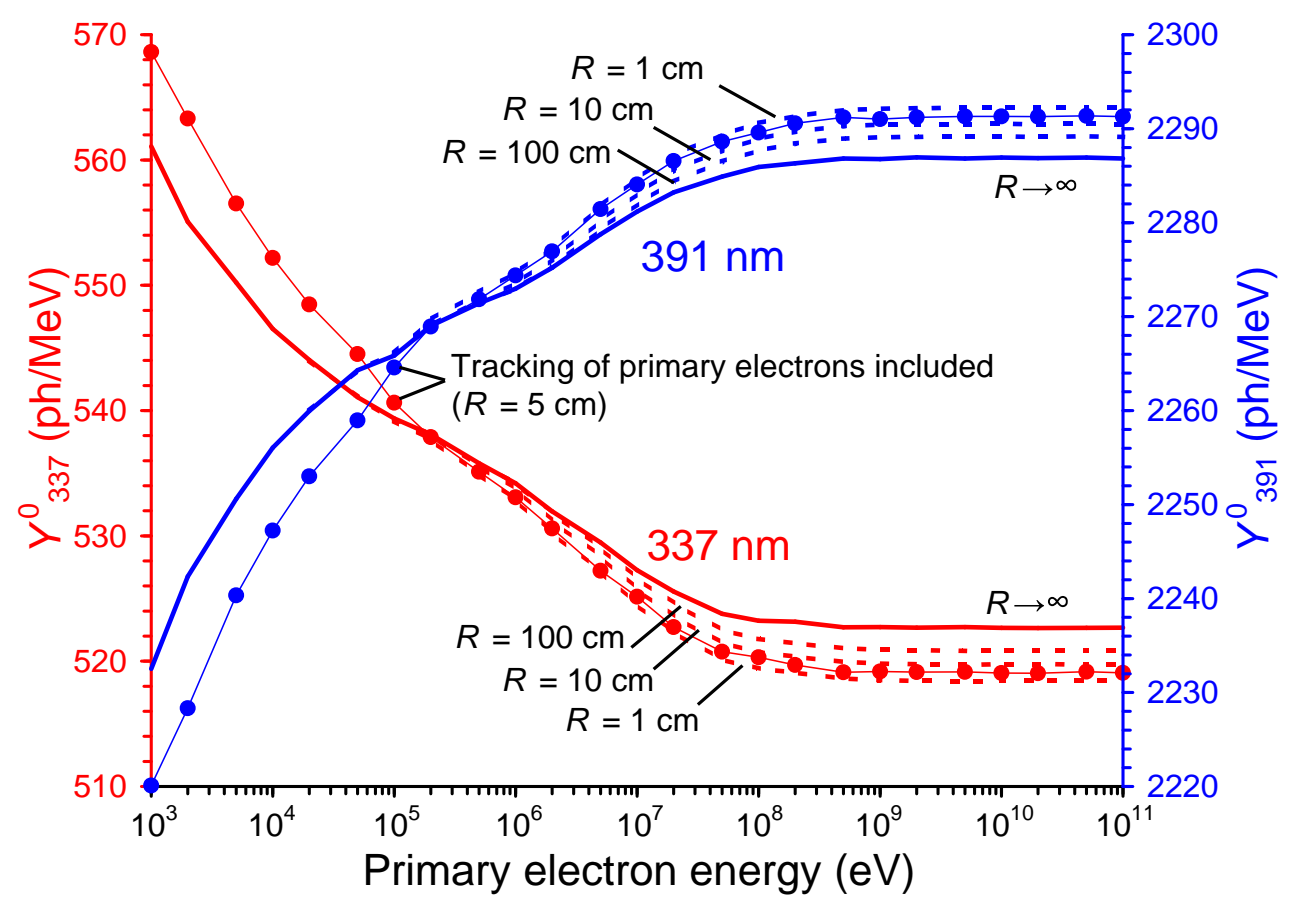

Figure 2: $\mathrm{MC}$ results of the FY in nitrogen at $1.137 \mathrm{mg} / \mathrm{cm}^{3}$ (i.e., $1 \mathrm{~atm}$ and $300 \mathrm{~K}$ ) in the absence of collisional quenching. Solid thick lines: FY at $337 \mathrm{~nm}$ (left axis, in red) and at $391 \mathrm{~nm}$ (right axis, in blue) obtained from the generic simulation for an infinite medium. Dotted lines: FY obtained from the generic simulation restricted to spheres of radii $R=1,10$ and $100 \mathrm{~cm}$. Circles connected with lines: FY calculated by tracking both primary and secondary electrons inside a sphere of radius $R=5 \mathrm{~cm}$.

the $337 \mathrm{~nm}$ band at $1 \mathrm{keV}$ is $9 \%$ greater than that at $100 \mathrm{MeV}$ according to this simulation.

Although the fluorescence emission in the $1 \mathrm{~N}$ system dominates at low pressure, it only represents a small fraction of the total fluorescence at atmospheric conditions owing to the strong collisional quenching of this band system. The $2 \mathrm{P}$ band at $337 \mathrm{~nm}$ is the most intense one at near atmospheric pressure; therefore, it is taken as the reference band for the analysis of the absolute FY in the following sections. 


\subsection{Theoretical value of the absolute air-fluorescence yield}

The FY for the $337 \mathrm{~nm}$ band in air at pressure $P$ is calculated from the following expression:

$$
Y_{337}=\frac{f_{\mathrm{N}_{2}} Y_{337}^{0}}{1+P / P_{337}^{\prime}},
$$

where $f_{\mathrm{N}_{2}}=0.78$ is the molecular fraction of $\mathrm{N}_{2}$ in air, $Y_{337}^{0}$ is the result of our simulation for nitrogen in the absence of quenching, and $P_{337}^{\prime}$ is the characteristic pressure accounting for collisional quenching in air.

In principle, the FY should be calculated from a simulation for air. However, the above expression is accurate enough for our purposes. In the first place, both fluorescence intensity and energy deposition per unit path length traveled by the incident electron are proportional to the amount of generated secondary electrons; thus, the small differences in the total ionization cross sections of nitrogen and air ${ }^{5}$ are almost exactly canceled out in the FY. Other features, like the slowdown of secondary electrons in these gases, may also affect the fluorescence production, but the differences in the inelastic cross sections of $\mathrm{N}_{2}$ and $\mathrm{O}_{2}$ at low energy are comparable to the uncertainties of available measurements [31, 32] and therefore they were disregarded in our calculations.

Our MC algorithm predicts $Y_{337}^{0} \approx 520 \mathrm{ph} / \mathrm{MeV}$ for high-energy primary electrons and a reasonably large interaction volume $(R \gtrsim 1 \mathrm{~cm})$. Using the $P_{337}^{\prime}$ value from [33] in expression (1), we obtained a theoretical value of $Y_{337}=7.9 \mathrm{ph} / \mathrm{MeV}$ at the reference conditions used in the following sections (i.e., dry air, $800 \mathrm{hPa}$ and $293 \mathrm{~K}$ ). The uncertainty of this result was estimated to be about $25 \%$, which is relatively large because of the contributions of many molecular parameters (e.g., the energy spectrum of secondary electrons, the stopping power at very low energy and the emission cross section). Efforts are currently being made to asses the error contributions and ultimately reduce the uncertainty of this theoretical value. Note, however, that this uncertainty in the absolute value does not apply to the weak dependencies of the FY on energy and the size of the interaction volume shown in figure 2 .

Some additional remarks are worth making. Our MC algorithm uses

\footnotetext{
${ }^{5}$ Using data from [30], we estimated that the average ionization cross section per air molecule is $3 \%$ larger than that of nitrogen at high energy. This is mostly due to the differences in the atomic numbers of nitrogen and oxygen (see footnote 3 ).
} 
experimental electron-impact emission cross sections that include possible contributions of cascade from upper lying excited states (see, e.g., [34]) $]^{6}$ On the other hand, more complicated excitations channels involving collisions with other molecules [5, 35, 36] were not included in our algorithm. Nevertheless, these processes can be neglected in air because the collisional quenching by $\mathrm{O}_{2}$ dominates. In addition, it has been proved in [33, 36] that the pressure dependence of the fluorescence intensity is well described by a simple Stern-Volmer law as that assumed in equation (1), where the effective $P^{\prime}$ value accounts for all the possible collisional processes affecting the fluorescence emission. Available measurements of the $P^{\prime}$ parameter for the band at $337 \mathrm{~nm}$ are in general agreement. Moreover, it was shown in [10, 21] that the discrepancies between the $P^{\prime}$ values reported by M. Nagano et al. [13] and the Airfly Collaboration [33] disappear when the measurements of M. Nagano et al. are properly corrected to account for $\delta$ rays leaving the experimental interaction volume.

A recent experimental study carried out by T. Dandl et al. [18] showed the presence of a long afterglow following pulsed excitation of pure nitrogen, which could be attributed to recombination processes of $\mathrm{N}_{2}^{+}$. This fluorescence contribution is strongly attenuated when the nitrogen gas has traces of impurities of $\mathrm{O}_{2}$ or other molecular species and it is negligible in air. Therefore, this effect has no impact on both the above theoretical result and the analysis of air showers detected by fluorescence telescopes.

\section{Critical analysis of absolute measurements of the fluorescence yield}

Since the nineties, several measurements of the absolute FY oriented to the cosmic-ray field have been carried out [12-19]. A summary of these measurements is given in table 1 .

Most experiments used electrons either from a ${ }^{90} \mathrm{Sr}$ radioactive source [12 15, 17] with an average energy of around $1 \mathrm{MeV}$, or from accelerators [12, 15, 16], which can provide energies in the GeV range. T. Dandl et al. [18] employed an electron gun producing a dc-beam of $\sim 10 \mathrm{keV}$ electrons,

\footnotetext{
${ }^{6}$ We showed in [10] that relative intensities of the fluorescence spectrum are proportional to the corresponding Franck-Condon factors for direct excitations of $\mathrm{N}_{2}$ from the ground state, indicating that cascade effects should be small.
} 


\begin{tabular}{lllll} 
Experiment & Particle & Energy $(\mathrm{MeV})$ & Wavelength interval $(\mathrm{nm})$ & Evaluation of $E_{\text {dep }}$ \\
\hline $\begin{array}{llll}\text { F. Kakimoto et al. [12] } \\
\text { M. Nagano et al. 13] }\end{array}$ & $\mathrm{e}^{-}$ & $1.4,300,600,1000$ & $337,300-400$ & No \\
G. Lefeuvre et al. [14] & $\mathrm{e}^{-}$ & 0.85 & 337 & No \\
MACFLY [15] & $\mathrm{e}^{-}$ & $1.1,1.5$ & $300-430$ & No \\
FLASH [16] & $\mathrm{e}^{-}$ & $2.85 \cdot 10^{4}$ & $300-420$ & Geant4 \\
AirLight [17] & $\mathrm{e}^{-}$ & $0.2-2$ & 337 & EGS4 \\
T. Dandl et al. [18] & $\mathrm{e}^{-}$ & $1 \cdot 10^{-2}$ & 337 & Geant4 \\
Airfly [19] & $\mathrm{p}$ & $1.2 \cdot 10^{5}$ & 337 & Not needed \\
\end{tabular}

${ }^{a}$ Electrons of $10 \mathrm{keV}$ stop completely in an air volume of about $1 \mathrm{~mm}^{3}$.

Table 1: Measurements of the absolute FY in air available in the literature. The type of particle, energy and wavelength interval used in each experiment are indicated. The MC code (if any) employed for the evaluation of the energy deposition is shown in the last column.

and Airfly [19] used $120 \mathrm{GeV}$ protons from the Test Beam Facility of the Fermi National Accelerator Laboratory.

Narrow-band filters were used in [12, 13, 17 19], providing the FY for the reference $2 \mathrm{P}$ band at $337 \mathrm{~nm}$. On the other hand, some measurements [12, 1416] were performed by using wide-band filters similar to those employed in fluorescence telescopes, which typically collect light in the 300-400 nm spectral range. For these measurements, we made a wavelength normalization to obtain the FY for the $337 \mathrm{~nm}$ band using precise experimental relative intensities from [33] 7 The estimated uncertainty associated with this normalization is $1.7 \%$, which was conservatively calculated by treating the systematic uncertainties of the relative intensities as partly correlated, as suggested by the authors.

In addition, all the results were normalized to common air conditions of $800 \mathrm{hPa}$ and $293 \mathrm{~K}$. If the pressure and temperature dependence was measured in a given experiment, these data were used for the normalization of the corresponding FY result. Otherwise, precise quenching data from [33, 37] were used. Nevertheless, the use of different possible $P^{\prime}$ values leads to deviations less than $1 \%$ in the FY values. More details on this normalization to common wavelength and air conditions are given in [11].

\footnotetext{
${ }^{7}$ Theoretical relationships of the relative intensities given in [10, in full agreement with experimental data, were also used to normalize the measurements of the MACFLY Collaboration [15], which include some weak bands outside the spectral range of [33.
} 

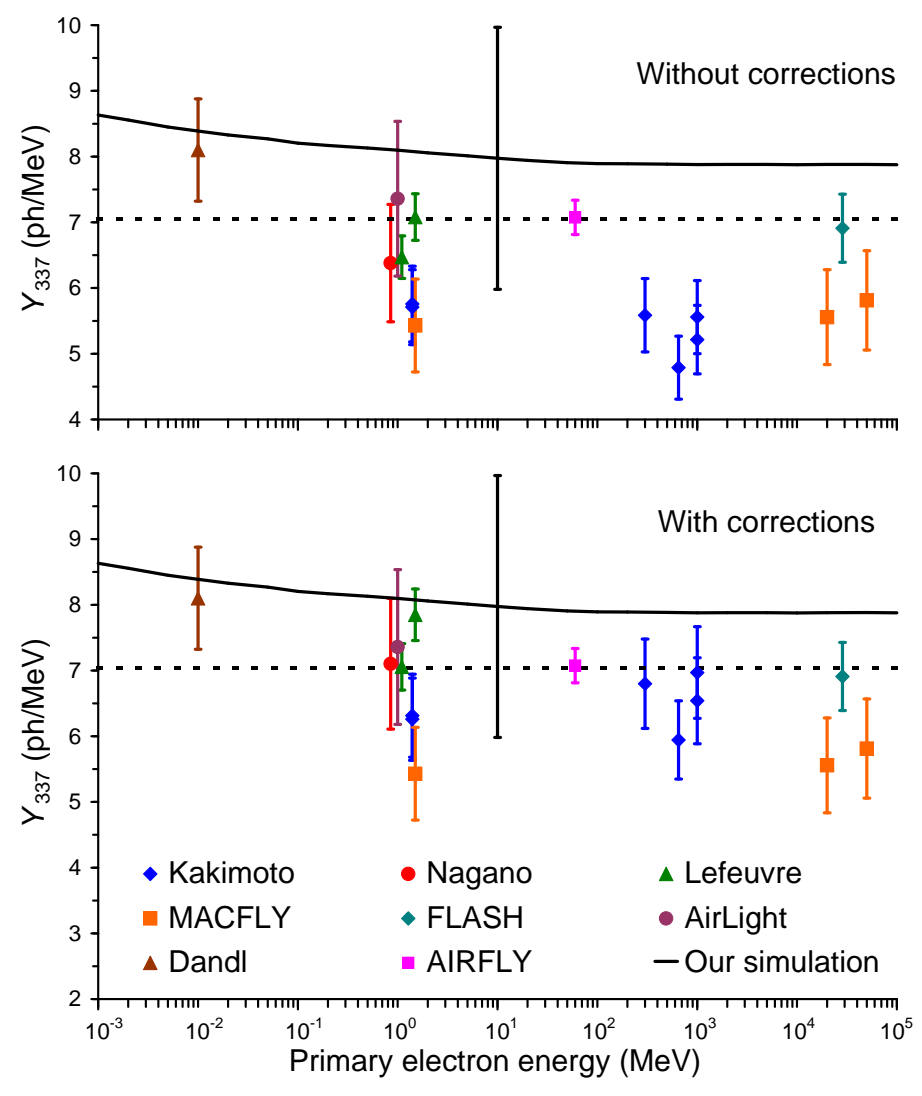

Figure 3: FY measurements normalized to common conditions $(337 \mathrm{~nm}$ band in air at $800 \mathrm{hPa}$ and $293 \mathrm{~K}$ ). Original results are shown in the top panel. Some measurements were corrected for a bias in the energy deposition in the bottom panel. The horizontal dashed line at $7.04 \mathrm{ph} / \mathrm{MeV}$ represents our average FY determined in section 5 . The solid line represents the theoretical prediction of the absolute FY from the MC simulation described in 4.2 with the corresponding estimated uncertainty (error bar plotted at $10 \mathrm{MeV}$ ).

The normalized FY values are plotted against the incident electron energy in the top panel of figure 3. An electron-equivalent energy of $60 \mathrm{MeV}$ was assigned to the Airfly measurement for $120 \mathrm{GeV}$ protons (see subsection 4.2. A general agreement is found, although discrepancies are larger than experimental error bars in some cases. The horizontal dashed line at $7.04 \mathrm{ph} / \mathrm{MeV}$ represents our average FY determined in section 5 .

In these experiments, an accurate calculation of the energy deposited in the field of view of the detector is required to determine the FY. In recent 
works [15-17, 19], the energy deposition was carefully evaluated by means of detailed Geant4 [28] or EGS4 [38] simulations. However, in other well-known experiments [12 14], the energy deposition was assumed to be equal to the electron energy loss calculated from the Bethe-Bloch formula, i.e., they neglected the energy carried away by $\delta$ rays that escape the detector field of view. Note that this assumption only affects the evaluation of the energy deposition, not the measurement of the fluorescence intensity. Therefore, the results of these experiments should be considered fully reliable if appropriate corrections are applied to the energy deposition. These corrections are described in subsection 4.1 and they lead to the scenario shown in the bottom panel of figure 3. Additional remarks on the evaluation of the energy deposition are given in that subsection too.

Effects of the possible dependence of the FY on the energy and type of incident particle are discussed in subsection 4.2. Other considerations on the use of synthetic air and pure nitrogen in these experiments are made in subsection 4.3 .

\subsection{Evaluation of the energy deposition}

As pointed out above, the FY values reported by F. Kakimoto et al. [12], M. Nagano et al. [13] and G. Lefeuvre et al. [14] have to be corrected to account for a bias in the energy deposition. In [11, we performed a dedicated simulation of the experiment of M. Nagano et al. including the geometrical details of their setup and other experimental features. We have redone this detailed simulation implementing the upgrades described above. The corresponding updated correction on the FY value of this experiment amounts to $+11 \%$. This result is fully consistent with the predictions from the generic simulation for a sphere of $5 \mathrm{~cm}$ radius that approximately accounts for the interaction region of this experiment.

Following the same procedure of [11], we obtained the corrections to the FY results of F. Kakimoto et al. and G. Lefeuvre et al. from the generic simulation for $R=10 \mathrm{~cm}$ and $R=4 \mathrm{~cm}$, respectively. For the last experiment, effects of electron scattering by the lead walls of the interaction chamber were also considered in our calculations (see [11] for details). As a result, the FY value of F. Kakimoto et al. at an average electron energy of $1.4 \mathrm{MeV}$ was increased by $10 \%$, and those of G. Lefeuvre et al. at $1.1 \mathrm{MeV}$ and $1.5 \mathrm{MeV}$ were increased by $9 \%$ and $11 \%$, respectively. Larger corrections, ranging from $+22 \%$ to $+25 \%$, were applied to the FY results of F. Kakimoto et al. 
for incident electrons of 300, 600 and $1000 \mathrm{MeV} \mathrm{B}^{8}$ As illustrated in figure 3 , our corrections lead to a better agreement of results.

In addition, we carried out in [11] detailed simulations of the experiments of MACFLY [15], FLASH [16] and AirLight [17], and they were compared with the simulations performed by the authors of these experiments. After including the upgrades in our MC algorithm, we found that our simulation results of energy deposition are in excellent agreement with those of MACFLY using Geant4 as well as with results of other Geant4 simulations that we performed independently [23]. On the other hand, the energy deposition determined by AirLight, also using Geant4, is 5\% lower than our prediction for this experiment. This could be justified by a coding error in the simulation of AirLight that the authors reported later in 39. If our simulation results were used, the FY value of AirLight should be lowered by $5 \%$. We also found discrepancies between the EGS4 simulation performed by FLASH and ours, which would lead to a correction of $+5 \%$ in their FY value. The origin of these discrepancies for the FLASH experiment is still unclear, but we observed a possible inconsistency in the treatment of density effect between both simulations (see [11, 22] for details).

Airfly assumed a $2 \%$ contribution to the total uncertainty of the FY due to the determination of the energy deposition by means of a Geant4 simulation. This uncertainty is consistent with our analysis reported in [23]. FLASH assumed a 1\% uncertainty in their EGS4 simulation. However, the remaining experiments neglected this error contribution in comparison with more relevant ones.

\subsection{Dependence on the energy and type of the incident particle}

According to our simulations, the FY for the $337 \mathrm{~nm}$ band and incident electron energies in the $\mathrm{keV}$ range could be up to $9 \%$ greater than that at high energies. In figure 3, we show the theoretical FY in air at the reference conditions obtained from a realistic simulation of an electron beam crossing a sphere of radius $R=5 \mathrm{~cm}$ (see subsection 3.2). The experimental data are compatible with this theoretical prediction of a weak energy dependence of the FY. In particular, the measurement of T. Dandl et al. [18] for $10 \mathrm{keV}$ electrons stopping in air is about $15 \%$ greater than those of other measure-

\footnotetext{
${ }^{8}$ These results at high energy were not considered to calculate our average FY, because F. Kakimoto et al. only used their measurement at $1.4 \mathrm{MeV}$ to gave their final result.
} 
ments at higher energies. The differences are however comparable to the experimental uncertainties; therefore, these results are not conclusive.

In principle, the FY might also depend on the type of the incident particle. So, the FY measured by Airfly [19] for $120 \mathrm{GeV}$ protons could be different to that for electrons, which is the one needed for the analysis of extensive air showers. Nevertheless, as discussed in Appendix A, the energy spectra of secondary electrons generated by incident electrons and protons of same velocity only differ at the highest $W$ values, and no significant effect on the FY is expected. To verify this, we implemented in the generic simulation described in section 3 the energy spectrum of secondary electrons produced by a proton of $120 \mathrm{GeV}$. The difference between the resulting FY and the one obtained for $60 \mathrm{MeV}$ electrons is well below $1 \%$.

\subsection{Effects related to gas composition}

The above experiments used synthetic air to ensure experimental conditions free from water vapor and pollutants. Synthetic air for research purposes is typically a $\mathrm{N}_{2}-\mathrm{O}_{2}$ mixture with a nitrogen fraction between $79 \%$ and $80 \%$, whereas the composition of atmospheric dry air is $78.08 \%$ of $\mathrm{N}_{2}$, $20.95 \%$ of $\mathrm{O}_{2}$ and $0.93 \%$ of $\mathrm{Ar}(<0.1 \%$ of others components). The mass stopping power and the electron trajectories are expected to be insensitive to the small differences between the compositions of synthetic air-like mixtures and atmospheric air. However, the effect on the measured FY may be significant, because the fluorescence production is proportional to the nitrogen fraction in the gas and it also depends on the concentration of the other components through the collisional quenching. Airfly used a $\mathrm{N}_{2}-\mathrm{O}_{2}$ mixture with $(79 \pm 1) \%$ of nitrogen and, consequently, added a $1 \%$ contribution to the total uncertainty due to this effect. On the other hand, most experiments neglected this error contribution.

To evaluate the impact of using synthetic air on the FY, we used the following expression:

$$
Y_{\text {air }}=Y_{\text {gas }} \frac{f_{\mathrm{N}_{2}}^{\text {air }}}{f_{\mathrm{N}_{2}}^{\text {gas }}} \frac{1+P / P_{\text {gas }}^{\prime}}{1+P / P_{\text {air }}^{\prime}},
$$

where $Y_{\text {gas }}$ is the FY measured in synthetic air at given pressure $P$ and $Y_{\text {air }}$ is the one in atmospheric dry air at same pressure. The characteristic pressure for a given $\mathrm{N}_{2}-\mathrm{O}_{2}$ mixture is determined from the partial $P^{\prime}$ values for the two components by using quenching data from [33]. According to this equation, 
the measurement of M. Nagano et al., who used a mixture of $78.8 \%$ of $\mathrm{N}_{2}$ and $21.2 \%$ of $\mathrm{O}_{2}$, should be reduced by about $1 \%$ to obtain the $\mathrm{FY}$ in dry air. In principle, other results should also be reduced by a similar amount, but precise data of the composition of the air-like mixtures used by these experiments are not explicitly given in some cases.

In the experiments of T. Dandl et al. and Airfly, the absolute measurement of the FY was actually performed in nitrogen (the fluorescence emission in air is strongly quenched by $\mathrm{O}_{2}$ ), and the authors converted it into the FY in air by using the ratio of the fluorescence intensity in nitrogen to that of air obtained in a separate measurement. However, the intensity ratio measured by T. Dandl et al. at near atmospheric pressure is about a factor of two higher than the ratio determined by Airfly in [19], which agrees with their previous measurement reported in [33] as well as with the measurement performed by M. Nagano et al. As pointed out in subsection 3.3, T. Dandl et al. showed indications of recombination processes that only take place in very pure nitrogen and that contribute with a long afterglow $(\sim 100 \mu \mathrm{s})$ to the total fluorescence. In view of that, they argued that the discrepancies in the intensity ratios may be due to a higher purity of the nitrogen gas that they used in their measurements. In principle, this would imply that the FY result of Airfly could be subjected to unknown uncertainties due to impurities in nitrogen. Nevertheless, the above discrepancies can be justified by the fact that T. Dandl et al. measured the continuous light induced by a dc-beam of electrons, whereas both M. Nagano et al. and Airfly registered fluorescence photons in coincidence with the arrival of single incident particles in a short interval of few tens of nanosecond, where the contribution from this long afterglow should be negligible. Moreover, in these coincidence measurements, any small contribution from this afterglow should be subtracted along with the continuous background. Therefore, we discard any uncertainty due to this effect in the Arfly result.

\section{Determination of an average fluorescence-yield value}

For the purpose of determining an average FY, we took the final result reported by each experiment, which corresponds to either a single measurement or a combination of several ones at different conditions (see table 1 and figure 3). As described above, all the results were normalized to common conditions (i.e., the $337 \mathrm{~nm}$ band in dry air at $800 \mathrm{hPa}$ and $293 \mathrm{~K}$ ) and those of F. Kakimoto et al., M. Nagano et al. and G. Lefeuvre et al. were also 


\begin{tabular}{lll} 
Experiment & $Y_{337}(\mathrm{ph} / \mathrm{MeV})$ & Uncertainty \\
\hline F. Kakimoto et al. [12] & 6.24 & $10 \%$ \\
M. Nagano et al. [13] & 7.10 & $14 \%$ \\
G. Lefeuvre et al. [14] & 7.46 & $5 \%$ \\
MACFLY [15] & 5.62 & $13 \%$ \\
FLASH [16] & 6.91 & $7.5 \%$ \\
AirLight [17] & 7.36 & $16 \%$ \\
T. Dandl et al. [18] & 8.10 & $10 \%$ \\
Airfly [19] & 7.07 & $4 \%$
\end{tabular}

Table 2: Normalized FY values and associated uncertainties (337 $\mathrm{nm}$ band in dry air at $800 \mathrm{hPa}$ and $293 \mathrm{~K}$ ). Corrections for the energy deposition were applied to the results of F. Kakimoto et al., M. Nagano et al. and G. Lefeuvre et al.

corrected for a bias due to the energy deposition. The normalized FY values and the uncertainties reported by the experiments are shown in table 2 and in figure 4. The original results of F. Kakimoto et al., M. Nagano et al. and G. Lefeuvre et al. are also shown in the figure (grey bars) to illustrate the effect of our corrections. Our average FY and its uncertainty interval are represented by vertical solid and dashed lines, respectively. The theoretical FY value determined in subsection 3.3 is shown in the figure too for comparison purposes.

The basic formulations used to compute the average FY and the associated uncertainty are described in subsection 5.1. Several consistency checks are also performed in this subsection. The impact on the average of the several experimental aspects discussed in section 4 is evaluated in subsection 5.2 to determine our final result.

\subsection{Formulations and consistency checks}

In principle, we may assume that the quoted experimental uncertainties represent the actual standard deviations of the corresponding (normal) probability distributions and that there is no correlation between experiments. Under this assumption, the best estimator of the FY would be

$$
\langle Y\rangle=\frac{\sum_{i=1}^{n} Y_{i} / \sigma_{i}^{2}}{\sum_{i=1}^{n} 1 / \sigma_{i}^{2}},
$$




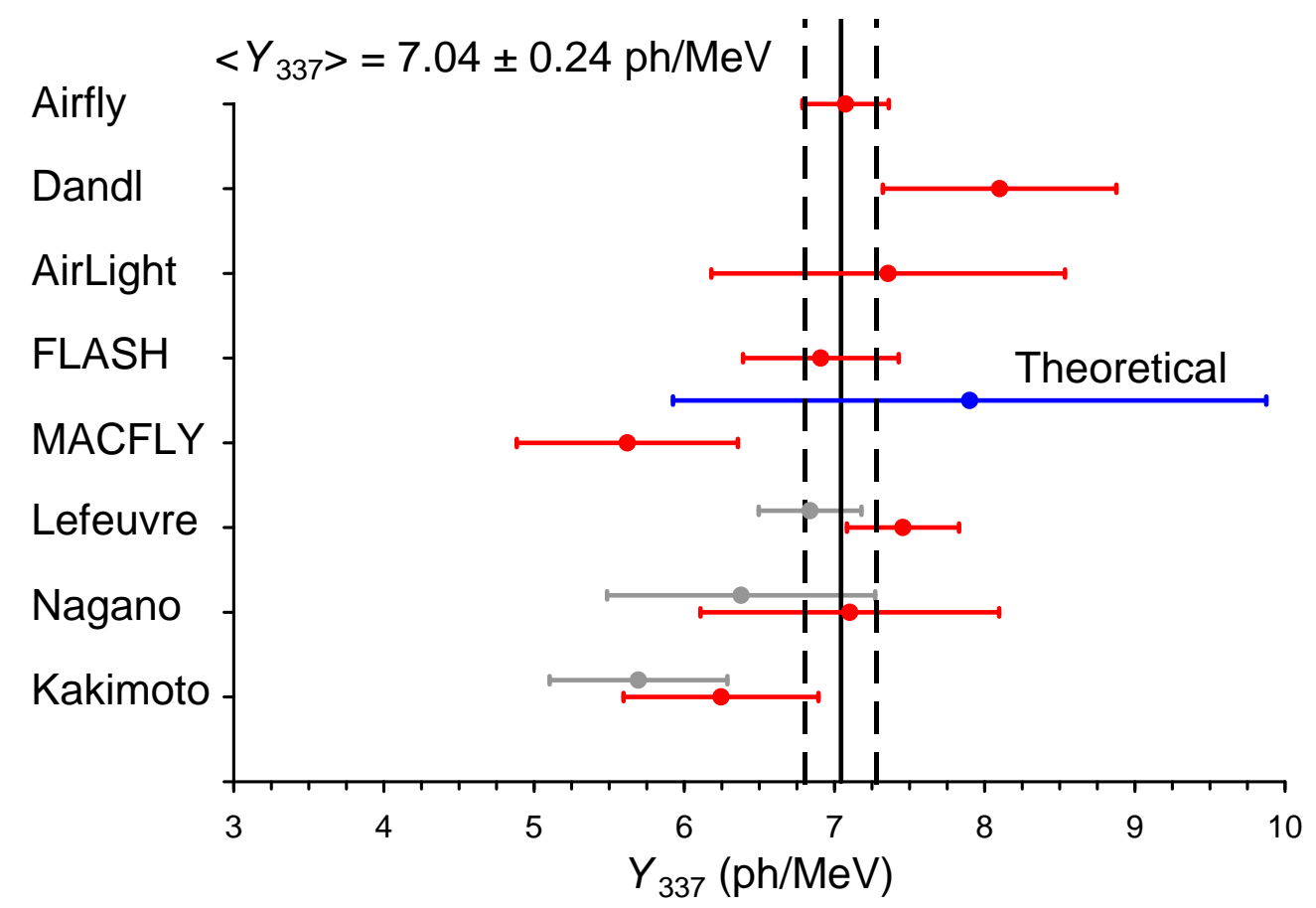

Figure 4: Graphical representation of the normalized FY values and associated uncertainties (see table 22). The original results of F. Kakimoto et al. [12, M. Nagano et al. [13] and G. Lefeuvre et al. [14] are also shown (grey bars) to illustrate the impact of our corrections. The solid and dashed vertical lines represent our final result of the average and uncertainty given in the uppermost label (see subsection 5.2). The blue bar represents the theoretical absolute FY predicted by our MC algorithm.

and the associated uncertainty would be given by

$$
\sigma_{\langle Y\rangle}^{2}=\frac{1}{\sum_{i=1}^{n} 1 / \sigma_{i}^{2}},
$$

where $Y_{i}$ and $\sigma_{i}$ are respectively the normalized FY value and the uncertainty of experiment $i$, and $n=8$ is the number of experiments. The $\chi^{2}$ statistic divided by the number of degrees of freedom (ndf) is defined in this case by

$$
\chi^{2} / \mathrm{ndf}=\frac{1}{n-1} \sum_{i=1}^{n} \frac{\left(Y_{i}-\langle Y\rangle\right)^{2}}{\sigma_{i}^{2}} .
$$

From the data given in table 2 , the above expressions yield an average of $7.06 \mathrm{ph} / \mathrm{MeV}$, a relative uncertainty of $2.6 \%$ and $\chi^{2} / \mathrm{ndf}=1.21$. The 
probability to obtain a larger $\chi^{2}$ value is $\alpha=0.29$, which is an acceptable value indicating that results are consistent with each other. However, the uncertainty of this average FY is surely not realistic, because the quoted experimental uncertainties may be underestimated in some cases and other possible effects discussed in section 4 can also affect these FY values. Although our final result was determined by a somewhat different procedure (see subsection 5.2), expressions (3 5) were used to perform several consistency checks of the data sample as described below.

We checked that, if a single measurement is removed from the sample, the average value varies by less than $\pm 1.7 \%$ with respect to that of the full sample. If two any measurements are removed instead, the average varies by less than $\pm 3.9 \%$. These deviations in the average are always consistent with the uncertainty calculated from equation (4) for the corresponding subsample, and the probability $\alpha$ associated with the $\chi^{2}$ value takes acceptable values ranging from 0.13 to 0.80 . This supports the consistency of results and suggests that the experimental uncertainties are not correlated.

Our corrections to the measurements of F. Kakimoto et al., M. Nagano et al. and G. Lefeuvre et al. eliminate a bias in the average. If these corrections were not included, an average value of $6.82 \mathrm{ph} / \mathrm{MeV}$ would be obtained. Note that this average is lower by an amount of $3.3 \%$, which is unacceptable at this level of accuracy reached in this analysis. In addition, the $\chi^{2} /$ ndf statistic of the sample without these corrections is $1.46(\alpha=0.18)$, which is larger than the value of $1.21(\alpha=0.29)$ obtained for the sample with corrections. This indicates that the compatibility of results improves when our corrections are applied. Alternatively, if these three measurements are removed from the sample to avoid any correction, an average of $7.00 \mathrm{ph} / \mathrm{MeV}$ is obtained with an associated uncertainty of $3.2 \%$ and $\chi^{2} / \mathrm{ndf}=1.42(\alpha=0.22)$. This result is fully compatible within uncertainties with the average for the whole sample with corrections.

We note that the above value of $7.06 \mathrm{ph} / \mathrm{MeV}$ and the result of Airfly are almost identical. This is partly due to the high precision of the Airfly measurement, which dominates the average. Nevertheless, even though this measurement were excluded from the data sample, a very close average of $7.04 \mathrm{ph} / \mathrm{MeV}$ would be obtained with an associated uncertainty as low as $3.3 \%$ and $\chi^{2} / \mathrm{ndf}=1.41(\alpha=0.21)$. Therefore, we can conclude that the result of Airfly is in full agreement with previous measurements and an average of the whole data sample provides a better precision. 


\subsection{Final result}

In a preliminary analysis [24], the FY result of $7.06 \mathrm{ph} / \mathrm{MeV}$ obtained directly by applying equation (3) was taken as the most likely mean value, and a simple procedure to evaluate a conservative uncertainty was followed. Here, we have employed a more rigorous method that leads to a very similar result. Several aspects previously discussed in section 4 were included in the present analysis:

1. We estimated an uncertainty of $\pm 2 \%$ in the evaluation of the energy deposition in all measurements except for that of T. Dandl et al.

2. The wavelength normalization of the FY results of F. Kakimoto et al., G. Lefeuvre et al., MACFLY and FLASH has an associated error contribution of $\pm 1.7 \%$.

3. The normalization to common pressure and temperature implies an error contribution that is conservatively estimated to be of $\pm 1 \%$.

4. The use of synthetic air in these experiments can lead to FY values systematically larger by about $1 \%$.

5. According to our simulation, the FY result of FLASH should be increased by $5 \%$ and that of AirLight should be decreased by $5 \%$.

6. If measurements are normalized to an electron energy of $100 \mathrm{MeV}$ according to the theoretical relative energy dependence predicted by our simulation, the FY results at around $1 \mathrm{MeV}$ of F. Kakimoto et al., M. Nagano et al., G. Lefeuvre et al. and AirLight should be decreased by $2-3 \%$ and that of T. Dandl et al. at $10 \mathrm{keV}$ should be decreased by $6 \%$.

The uncertainties mentioned in points 1-3 should show some degree of correlation between experiments. Note, for instance, that the corrections that we applied to the FY values of F. Kakimoto et al., M. Nagano et al. and G. Lefeuvre et al. are all evaluated with the same $\mathrm{MC}$ algorithm, and that measurements are typically carried out at an air pressure higher than $800 \mathrm{hPa}$. To be conservative, we made the extreme assumption that these systematic uncertainties are fully correlated.

For the points 4-6, instead of applying the proposed corrections, we conservatively treated them as additional uncertainty components (either positive or negative). For instance, point 5 was accounted for by considering an uncertainty components of $+5 \%$ and $-5 \%$ on the FY results of FLASH and AirLight, respectively. For the points 4 and 6, we assumed that the corresponding uncertainties are fully correlated between experiments (e.g., the systematic deviation of $1 \%$ in the FY due to the use of synthetic air affects 


\begin{tabular}{lll} 
Error source & $-\Delta\left\langle Y_{337}\right\rangle$ & $+\Delta\left\langle Y_{337}\right\rangle$ \\
\hline Energy deposition & $1.9 \%$ & $1.9 \%$ \\
Wavelength normalization & $0.7 \%$ & $0.7 \%$ \\
Pressure and temperature normalization & $1 \%$ & $1 \%$ \\
Air composition & $1 \%$ & - \\
Corrections to the results of FLASH and AirLight & $0.1 \%$ & $0.5 \%$ \\
Energy normalization & $1.1 \%$ & - \\
\hline Quadratic sum & $2.7 \%$ & $2.3 \%$
\end{tabular}

Table 3: Impact on the average of the error sources indicated in the points 1-6 of subsection 5.2. The negative and positive relative shifts with respect to the value of $7.06 \mathrm{ph} / \mathrm{MeV}$ are shown in columns 2 and 3. The quadratic sums of these error contributions are given in the last row.

either all of the measurements or none of them). Note that this treatment involves asymmetric uncertainties.

The procedure was as follows. First, the average and the associated uncertainty were calculated from equations (3) and (4) using only the uncorrelated part of the experimental uncertainties. In particular, the error contributions due to the energy deposition and the air composition included in the total uncertainties reported by FLASH and Airfly were removed in this step. The resulting average is still $7.06 \mathrm{ph} / \mathrm{MeV}$ and the corresponding (partial) uncertainty is $\pm 2.4 \%$. Then, for each error source $1-6$, we recalculated the average after modifying the pertinent FY values in their full uncertainty interval. The corresponding relative shifts of the average with respect to the above value of $7.06 \mathrm{ph} / \mathrm{MeV}$ are listed in table 3. All the relative shifts of same sign were added quadratically. As a result, we obtained an asymmetric systematic uncertainty interval of ${ }_{-2.7 \%}^{+2.3 \%}$ around $7.06 \mathrm{ph} / \mathrm{MeV}$, which was converted into a symmetric interval of $\pm 2.5 \%$ around $7.04 \mathrm{ph} / \mathrm{MeV}$. Finally, this systematic uncertainty was added quadratically to the one previously calculated from the uncorrelated uncertainties, resulting in a total uncertainty of $3.5 \%$. Therefore, our final result is $\left\langle Y_{337}\right\rangle=7.04 \pm 0.24 \mathrm{ph} / \mathrm{MeV}$ in dry air at $800 \mathrm{hPa}$ and $293 \mathrm{~K}$ (see figure 4 ).

\section{Conclusions}

The problem of the absolute value of the air-fluorescence yield was studied in depth by means of both a detailed MC simulation and a critical analysis 
of available measurements of the FY.

We carried out a theoretical evaluation of the absolute FY based on an upgraded MC algorithm that enables the calculation of the energy deposition and the fluorescence emission upon the passage of an energetic electron through a nitrogen volume of given geometry. The algorithm simulates all the individual interactions of both the incident electrons and the secondary particles (i.e., electrons and X rays) generated in the medium. Special care was paid in describing correctly the production of low-energy secondary electrons, which are the main responsible for the fluorescence emission. We obtained that the FY is independent of the incident electron energy (within 3\%) for energies above $1 \mathrm{MeV}$, whereas a weak energy dependence was found in the keV region. From our updated simulation results, we obtained a theoretical absolute $\mathrm{FY}$ value of $7.9 \pm 2.0 \mathrm{ph} / \mathrm{MeV}$ for the band at $337 \mathrm{~nm}$ in dry air at $800 \mathrm{hPa}$ and $293 \mathrm{~K}$, which is in good agreement with experimental values.

We analyzed the available measurements of the absolute FY considering many aspects. In particular, the evaluation of the energy deposited in the experimental interaction chamber was proved to be crucial for an accurate determination of the FY. Our simulation results of energy deposition were used to apply corrections to the FY results of some experiments that neglected the contribution of $\delta$ rays that leave the field of view of the detector measuring the fluorescence light. Other possible effects (e.g., the weak energy dependence predicted by our MC algorithm and the differences between the atmospheric air and the synthetic air-like mixtures used in these experiments) were also shown to be significant. As a final result of this analysis, we obtained an average absolute FY value of $7.04 \pm 0.24 \mathrm{ph} / \mathrm{MeV}$ at the reference conditions (i.e., $337 \mathrm{~nm}$ band, dry air at $800 \mathrm{hPa}$ and $293 \mathrm{~K}$ ). This result is very similar to the one recently reported by the Airfly Collaboration [19], but with a slight improvement in precision as a consequence of the consistency of all the FY measurements.

\section{Acknowledgement}

This work was supported by MINECO (FPA2009-07772, FPA2012-39489-

C04-02) and CONSOLIDER CPAN CSD2007-42. We thank our colleagues of the Auger Collaboration for fruitful discussions and comments on this work. 


\section{Appendix A. Energy spectrum of secondary electrons}

In the present upgraded version of the $\mathrm{MC}$ algorithm, the energy spectrum of secondary electrons generated by low incident electrons $(T<710 \mathrm{eV})$ is described by

$$
\frac{\mathrm{d} \sigma_{\text {ion }}}{\mathrm{d} W}=K_{\text {low }}\left[\frac{1}{w^{2}+W^{2}}+\frac{1}{w^{2}+(T-I-W)^{2}}\right] .
$$

This is the same empirical formula originally derived by [40] that was used in previous versions of our algorithm, but with the addition of an exchange term that is only relevant near the cutoff energy of the spectrum. Here, $K_{\text {low }}$ is a normalization factor (see below), $w$ is an adjustable parameter that is set equal to $11.4 \mathrm{eV}$ for $\mathrm{N}_{2}$, and $I=15.6 \mathrm{eV}$ is the ionization potential. The fastest of the two free electrons after an ionization collision is customarily defined as the primary (incident) one; therefore, the maximum energy of the secondary electron is $(T-I) / 2 ?^{9}$

For $T>710 \mathrm{eV}$, we followed the same procedure of [10], but incorporating exchange terms. An analytical expression of $\mathrm{d} \sigma_{\text {ion }} / \mathrm{d} W$ was constructed so that it approaches expression (A.1) at low $W$ and reproduces the exact Møller formula for electron-electron scattering cross section [41] in the high- $W$ limit. Also, this expression was adapted to be finite at $W=0$ and symmetric under the change $W \rightarrow T-I-W$. The result is

$$
\begin{aligned}
\frac{\mathrm{d} \sigma_{\text {ion }}}{\mathrm{d} W}= & \frac{4 \pi Z a_{0}^{2} \alpha^{2} R}{\beta^{2}}\left\{\left[1+K_{\text {high }} \exp \left(-\frac{W}{W_{\mathrm{t}}}\right)\right]\right. \\
& {\left[\frac{1}{w^{2}+W^{2}}+\frac{1}{w^{2}+(T-I-W)^{2}}\right] } \\
& \left.-\frac{2 \gamma-1}{\gamma^{2}(W+I / 2)(T-W-I / 2)}+\frac{1}{\left(\gamma m c^{2}\right)^{2}}\right\} .
\end{aligned}
$$

where $Z=14$ for molecular nitrogen, $a_{0}$ is the Bohr radius, $\alpha$ is the finestructure constant, $R$ is the Rydberg energy, $\beta$ is the ratio of the incident electron speed to the speed of light $c, \gamma$ is the Lorentz factor, and $m$ is the

\footnotetext{
${ }^{9}$ In our MC algorithm, an average excitation energy of $\left\langle E_{\text {exc }}^{\text {ion }}\right\rangle=1.3 \mathrm{eV}$ is assumed to be transferred to the molecule in every ionization event [10. Consequently, the parameter $I$ is in practice replaced with $I+\left\langle E_{\mathrm{exc}}^{\mathrm{ion}}\right\rangle=16.9 \mathrm{eV}$, and the maximum energy of secondary electrons is reduced accordingly for a correct energy balance.
} 
electron rest mass. The transition energy $W_{\mathrm{t}}=71 \mathrm{eV}$ was tuned so that the Bethe-Bloch stopping power is correctly reproduced (see section 3.1), and the $T$ value at which the transition from A.1 to A.2 takes place was chosen to provide a smooth join between both expressions.

The factors $K_{\text {low }}$ and $K_{\text {high }}$ in the above equations are determined by the normalization condition

$$
\sigma_{\text {ion }}=\int \mathrm{d} W \frac{\mathrm{d} \sigma_{\text {ion }}}{\mathrm{d} W}
$$

where $\sigma_{\text {ion }}$ is the total ionization cross section, which includes the densityeffect correction at high energy [8, 21].

As shown in figure A.5, results of equations A.1 and (A.2) are in good agreement with available experimental data [42, 43] in the corresponding $T$ domains, and they reproduce correctly the flatter behavior of the spectra near the cutoff energy as a consequence of exchange effects.

Our model assumes an structureless form A.1 at low $W$ that does not account for the fine details in the shape of the experimental spectra. This may be very relevant for a theoretical determination of the FY due to the strong energy dependence of the emission cross sections in the $10-100 \mathrm{eV}$ range [31. We are studying the use of more sophisticated models of the energy distribution of secondary electrons (see, e.g., [44]). Preliminary tests have indicated us that our approximation may imply a systematic error of up to $10 \%$ on the absolute FY value given in subsection 3.3 , for which we estimated a total estimated uncertainty of $25 \%$. In contrast, ignoring these details in the low- $W$ portion of the spectrum pose no impact on our results of energy deposition (subsection 3.1), because the accuracy of this parameter is basically limited by the uncertainties in the generation and transport of $\delta$ rays.

Analytical expressions for the energy spectrum of secondary electrons generated by incident particles other than electrons can be derived in a similar way. In fast collisions for which the first Born approximation is valid, the scattering cross section is proportional to the square of the projectile charge, that is, it is independent of the sign of charge. In addition, an important conclusion of the Bethe theory is that the cross section is basically independent of the projectile mass. As a consequence, both the integrated and differential ionization cross sections are almost identical for fast electrons and protons of same velocity. Differences in the energy spectra of secondary electrons generated by these two particles only arise near the cutoff energy, 


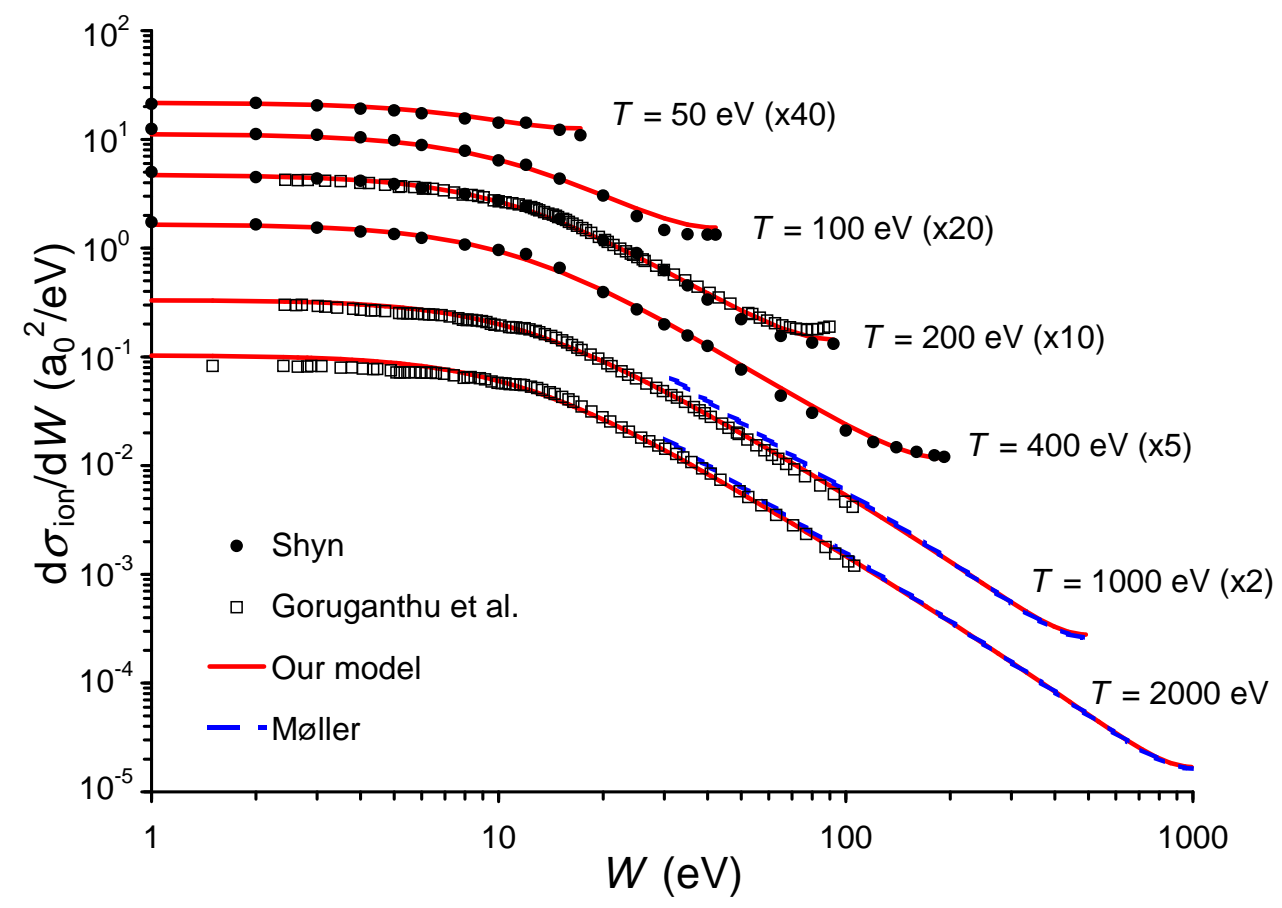

Figure A.5: Comparison of our model of the energy spectrum of secondary electrons with experimental data. Filled circles: experimental data of [42]. Empty squares: experimental data of [43. Solid lines (red): results of expressions (A.1) and (A.2) in their corresponding $T$ domains. Dashed lines (blue): Møller cross section.

which is obviously dependent on the projectile mas ${ }^{10}$ and where exchange effects (present in electron-electron scattering, but not in proton-electron scattering) are important.

\section{Appendix B. Angular distributions}

\section{Appendix B.1. Ionization collisions}

Let us assume a binary collision of an incident electron of kinetic energy $T$ with a target electron initially at rest in the laboratory frame. After the

\footnotetext{
${ }^{10}$ Conservation of energy and momentum restricts the maximum energy transferred by a proton to a free electron at rest to $4 T$, where $T$ is the kinetic energy of an electron with the same velocity as the proton.
} 
collision, the incident electron is scattered with an energy $T^{\prime}$ at a polar angle $\theta$ and an azimuthal angle $\phi$. The target electron recoils with an energy $W$ at polar angle $\theta_{\mathrm{s}}$ and azimuthal angle $\phi_{\mathrm{s}}=\phi+\pi$. Due to the axial symmetry about the direction of incidence, $\phi$ is a uniform random variable in the interval $(0,2 \pi)$. Once the scattering plane is defined, the kinematics of the collision is entirely determined by energy and momentum conservation.

On the other hand, in an ionization collision, the incident electron also transfers energy and momentum to the atom or molecule, and orbital electrons are not at rest, but move with a certain momentum distribution. In our MC algorithm, the energy transfer $T-T^{\prime}$ is assumed to be $W+I$ in outer-shell ionizations and $W+I_{\mathrm{K}}$ in K-shell ionizations, where $I_{\mathrm{K}}=410 \mathrm{eV}$ is the ionization potential of the $\mathrm{K}$ shell of nitrogen. The directions of motion of the scattered and ejected electrons are determined by assuming a binary collision, that is, neglecting both the momentum transferred to the molecule and the initial momentum of the target electron. In principle, this approximation is only valid in close collisions with large momentum transfer (see, e.g., [45]). Nevertheless, we can use it, with no lack of accuracy for our purposes, also in distant ionization collisions of high-energy electrons, where the momentum transfer is small and therefore $\theta \approx 0$. The angular distributions of scattered and ejected electrons of low energy cannot be described by this procedure; however, we applied it for all energies because the tracking of low-energy electrons has no relevant impact on the final results at near atmospheric conditions.

As an improvement only appreciable at low-pressure conditions, an angular straggling is introduced in the generation of secondary electrons. Experimental data of the angular distribution [43. can be approximately described by a Lorentzian function, where the mean value $\left\langle\theta_{\mathrm{s}}\right\rangle$ coincides with the angle corresponding to a binary collision and the width of the distribution decreases with $W$. From a fit to experimental data, we found that $\theta$ can be sampled from

$$
\theta_{\mathrm{s}}=\left\langle\theta_{\mathrm{s}}\right\rangle+\frac{0.98}{1+W / 65} \tan \left[\frac{\pi}{2}(2 r-1)\right]
$$

where $W$ is given in $\mathrm{eV}$ and $r$ is a random number in the interval $(0,1)$. Note that $\theta=\left\langle\theta_{\mathrm{s}}\right\rangle$ in the high- $W$ limit, as expected.

\section{Appendix B.2. Elastic collisions}

Theoretical calculations of both the integral and differential elastic cross sections of nitrogen from [46, 47] are used in our MC algorithm. Numeri- 
cal data of the total elastic cross section $\sigma_{\text {el }}$ are included in a database file, and values at given energy are obtained by log-log interpolation. For numerical purposes, $\theta$ values are efficiently sampled by using an approximated parameterization of the normalized angular distribution

$$
P(\theta)=\frac{\sin \theta}{2 \pi \sigma_{\mathrm{el}}} \frac{\mathrm{d} \sigma_{\mathrm{el}}}{\mathrm{d} \Omega} .
$$

Again, $\phi$ is distributed uniformly.

In the present version of our MC algorithm, the model of $P(\theta)$ was revised to achieve a better agreement with these theoretical calculations. The following ansatz was assumed:

$$
\begin{aligned}
P(\theta)= & (1-K) \frac{\left(1+a_{1}\right) \sin \theta}{\left.2\left[1+a_{1} \sin ^{2}(\theta / 2)\right)\right]^{2}} \\
& +K \frac{\left(1+a_{2}\right) \sin \theta}{\left.2\left[1+a_{2} \cos ^{2}(\theta / 2)\right)\right]^{2}} .
\end{aligned}
$$

The first (dominant) term of the right-hand side of this equation has the form corresponding to electron scattering by a Yukawa potential in the Born approximation, and the second (symmetric) term was introduced to account for the relatively large probability of backscattering at low energies. Then, we searched for suitable expressions for the dimensionless parameters $K, a_{1}$ and $a_{2}$ that fit theoretical data from [46, 47].

For $T<40 \mathrm{eV}$, we found ( $T$ expressed in $\mathrm{eV})$ :

$$
\begin{aligned}
& K=0.4458\left(1-\mathrm{e}^{-T / 33.98}\right) \\
& a_{1}=0.4062 \frac{T}{6.384}\left(1+\frac{T}{6.384}\right) \\
& a_{2}=9.658\left(1+\frac{T}{354.4}\right) .
\end{aligned}
$$

For $40 \mathrm{eV} \leq T<700 \mathrm{eV}$ :

$$
\begin{aligned}
K & =0.7876\left(1+\frac{T}{23.22}\right) \\
a_{1} & =1.475 T^{0.7018} \\
a_{2} & =9.210 \mathrm{e}^{-T / 135.3} .
\end{aligned}
$$


And for $T \geq 700 \mathrm{eV}$ :

$$
\begin{aligned}
& K=0 \\
& a_{1}=18.1\left(1+\frac{T^{2} / 2 m c^{2}+T}{121.7}\right) \\
& a_{2}=0 .
\end{aligned}
$$

\section{References}

[1] T. Abu-Zayyad et al., Nucl. Instrum. Meth. A 450 (2000) 253.

[2] J. Abraham et al., Nucl. Instr. Meth. A 620 (2010) 227.

[3] H. Tokuno et al., Nucl. Instr. Meth. A 676 (2012) 54.

[4] F. Kajino et al., 33rd International Cosmic-Ray Conference (Rio de Janeiro, 2013), paper 1128.

[5] A.E. Grün and E. Schopper, Z. Naturforsch A 9 (1954) 134;

A.E. Grün, Can. J. Phys. 36 (1958) 858.

[6] O. Stern and M. Volmer, Physik. Zeitschrift, 20 (1919) 183.

[7] F. Blanco and F. Arqueros, Phys. Lett. A 345 (2005) 355.

[8] F. Arqueros et al., Astropart. Phys. 26 (2006) 231.

[9] F. Arqueros, F. Blanco and J. Rosado, Nucl. Instrum. Meth. A 597 (2008) 94.

[10] F. Arqueros, F. Blanco and J. Rosado, New J. Phys. 11 (2009) 065011.

[11] J. Rosado, F. Blanco and F. Arqueros, Astropart. Phys. 34 (2010) 164.

[12] F. Kakimoto et al., Nucl. Instrum. Meth. A 372 (1996) 205.

[13] M. Nagano et al., Astropart. Phys. 22 (2004) 235.

[14] G. Lefeuvre et al., Nucl. Instrum. Meth. A 578 (2007) 78.

[15] P. Colin et al. [MACFLY Collaboration], Astropart. Phys. 27 (2007) 317. 
[16] R. Abbasi et al. [FLASH Collaboration], Astropart. Phys. 29 (2008) 77.

[17] T. Waldenmaier et al., Astropart. Phys. 29 (2008) 205.

[18] T. Dandl, T. Heindl and A. Ulrich, JINST 7 (2012) P11005.

[19] M. Ave et al. [AIRFLY Collaboration], Astropart. Phys. 42 (2013) 90.

[20] J. Rosado, F. Blanco and F. Arqueros, Average value of available measurements of the absolute air-fluorescence yield, unpublished manuscript. Available at http://arxiv.org/abs/1103.2022.

[21] J. Rosado, Analysis of the air fluorescence induced by electrons for application to cosmic-ray detection, $\mathrm{PhD}$ Thesis, Universidad Complutense de Madrid, 2011.

[22] J. Rosado, F. Blanco and F. Arqueros, AIP Conf. Proc. 1367 (2011) 34.

[23] J. Rosado et al., EPJ Conf. 53 (2013) 10001. Available at http://arxiv.org/abs/1207.2913.

[24] J. Rosado and F. Arqueros, 33rd International Cosmic-Ray Conference (Rio de Janeiro, 2013), paper 377.

[25] R.M. Sternheimer, Phys. Rev. 88 (1952) 851.

[26] J. Rosado et al., Nucl. Instrum. Meth. A 597 (2008) 83.

[27] A. Allisy et al., Stopping Powers for Electrons and Positrons (ICRU Report No. 37, 1984);

M.J. Berger et al., ESTAR: Computer Programs for Calculating Stopping-Power and Range Tables for Electrons (ver. 1.2.3, 2005, NIST). Available at http://physics.nist.gov/Star.

[28] S. Agostinelli et al., Nucl. Instrum. Meth. A 506 (2003) 250.

[29] M. Risse and D. Heck, Astropart. Phys. 20 (2004) 661.

[30] Y.-K. Kim et al., Electron impact cross sections for ionization and excitation (ver. 3.0, 2004, NIST). Available at http://physics.nist.gov/ionxsec.

[31] Y. Itikawa, J. Phys. Chem. Ref. Data 35 (2006) 31. 
[32] Y. Itikawa et al., J. Phys. Chem. Ref. Data 18 (1989) 23.

[33] M. Ave et al. [AIRFLY Collaboration], Astropart. Phys. 28 (2007) 41.

[34] J.T. Fons, R.S. Schappe and C.C. Lin, Phys. Rev. A 53 (1996) 2239.

[35] G. Dilecce, P.F. Ambrico and S. De Benedictis, Chem. Phys. Lett. 431 (2006) 241.

[36] A. Morozov et al., Eur. Phys. J. D 46 (2008) 51; A. Morozov et al., Nucl. Instr. Meth. A 597 (2008) 105.

[37] M. Ave et al. [AIRFLY Collaboration], Nucl. Instrum. Meth. A 597 (2008) 50 .

[38] W.R. Nelson, H. Hiragrama and D.W.O. Rogers, The EGS4 Code System (Stanford Linear Accelerator Center, SLAC-265, 1985).

[39] T. Waldenmaier et al., 8th Air-Fluorescence Workshop (Karlsruhe, 2011). Available at http://www.kceta.kit.edu/downloads/Talk_Waldenmaier.pdf

[40] C.B. Opal, E.C. Beaty and W.K. Peterson, At. Data 4 (1972) 209.

[41] V.B. Berestetskii, E.M. Lifshitz and L.P. Pitaevskii, Relativistic Quantum Theory (vol. 4, part 1 of A Course of Theoretical Physics), Pergamon Press (1971).

[42] T.W. Shyn, Phys. Rev. A 27 (1983) 2388.

[43] R.R. Goruganthu, W.G. Wilson and R.A. Bonham, Phys. Rev. A 35 (1987) 540 .

[44] Y.-K. Kim, J.P. Santos and F. Parente, Phys. Rev. A 62 (2000) 052710.

[45] J.M. Fernández-Varea et al., Nucl. Instrum. Meth. B 229 (2005) 187.

[46] F. Blanco and G. García, Phys. Lett. A 317 (2003) 458.

[47] A. Roldán et al., J. Appl. Phys. 95 (2004) 5868. 